\title{
Multilayer Coplanar Waveguide Transmission Lines Compatible with Standard Digital Silicon Technologies
}

\author{
Yunliang Zhu, Shang Wang, and Hui Wu \\ Laboratory for Advanced Integrated Circuits and Systems (LAICS) \\ Department of Electrical and Computer Engineering, University of Rochester \\ Rochester, NY 14627, Email: hwu@ece.rochester.edu
}

\begin{abstract}
On-chip transmission lines in silicon technologies suffer from the low-resistivity substrate and geometry limitations imposed by layout and metal density design rules. In this paper, we demonstrate that multilayer coplanar waveguide (MCPW) transmission lines can be utilized to overcome both problems by taking advantage of multiple metal layers available. We studied the effects of ground spacing on MCPW characteristics using electromagnetic simulations, based on process parameters from a $0.18 \mu \mathrm{m}$ standard digital CMOS technology with $0.01 \Omega-\mathrm{cm} \mathrm{P}+$ substrate. Simulation results show that by adjusting the ground spacing, we can control the characteristics impedance, effective dielectric constant, and attenuation of MCPW lines. A test chip was designed and fabricated with MCPW, regular CPW, and microstrip lines. Measurement results verified the analysis and simulation results.
\end{abstract}

\section{INTRODUCTION}

Silicon technologies, such as deep-submicron CMOS and $\mathrm{SiGe}$ BiCMOS, have found increasingly wide adoption in microwave and millimeter-wave integrated circuits. This is driven by their advantages of low cost and system-on-a-chip capabilities, and enabled by rapid advances in the transistor performance. For example, the cut-off frequency $\left(f_{T}\right)$ of NMOS transistors is over $150 \mathrm{GHz}$ in $90 \mathrm{~nm} \mathrm{CMOS}$, and can go beyond $200 \mathrm{GHz}$ at the $65 \mathrm{~nm}$ node [1]. Another important trend is the reverse scaling of metal layers in silicon technologies, as shown in Fig. 1. Firstly, more metal layers are added in each technology generation. Driven by increasing current-carrying requirement in global interconnects such as power and clock distribution networks, top metal layers are also made thicker. Both factors increase the distance of top metal layers to silicon substrate. Thicker metals reduce conductor loss, and larger metal-to-substrate distance decreases parasitic capacitance and dielectric loss. These changes in metals have significantly improved the performance of on-chip transmission lines in silicon, which not only benefits highspeed interconnects [2], but also enables constructing passive devices for microwave and millimeter-wave applications [3].

Despite these progresses, however, silicon technologies still pose serious challenges for on-chip transmission lines. First, low-resistivity silicon substrate $(\sim 0.01 \Omega \cdot \mathrm{cm})$ commonly used in silicon technologies to combat the latch-up problem causes severe loss in on-chip transmission lines through both capacitive and magnetic coupling. Change to substrate with higher resistivity $(\sim 10 \Omega \cdot \mathrm{cm})$ only alleviates the problem at extra cost and lower yield, and further increase of substrate resistivity is unlikely. A high-resistivity layer close to the wafer

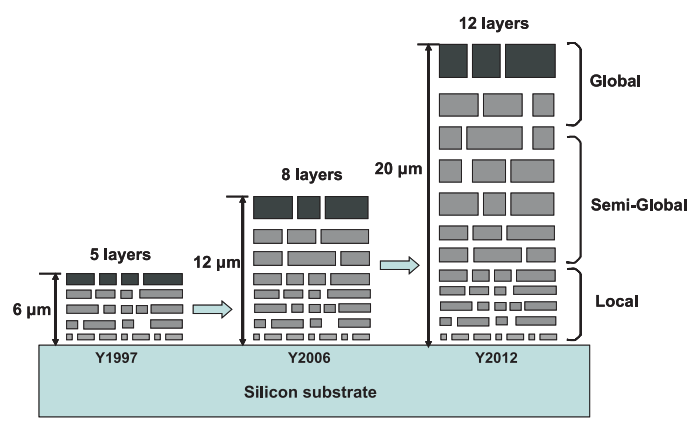

Fig. 1. Reverse scaling for top metals in CMOS technologies (not to scale, dielectric not shown), according to ITRS roadmap [1].

surface, such as that in silicon-on-insulator (SOI) technologies, is usually too thin to be effective in reducing substrate loss. A second limitation for on-chip transmission lines in silicon is due to layout design rules on thick top metal layers. Metalpatterning damascene processes in silicon technologies result in the aspect ratio of metal structures to be quite small (usually less than one). So metal width and spacing for thick top metals need to be at least several microns typically. The specified minimum metal width sets the upper limit on the characteristic impedance, and the minimum metal spacing usually is not optimal for low-loss transmission lines. The third limitation of silicon technologies comes as the side effect of multiple metal layers. Chemical-mechanical polishing (CMP) processes are used to achieve flat surface after each metal-patterning step. So there is a minimum metal density requirement for each metal layer in order to avoid uniformity problems in CMP. This means all metal layers need to be filled to the required density according to the design rule, using dummy metal structures if necessary. Since most on-chip transmission lines are built on top metal layers, the metal fills in the layers underneath effectively reduce the dielectric constant of the inter-layer dielectric materials (mostly silicon dioxide), and result in larger parasitic capacitance, which in turn results in lower impedance and larger substrate loss.

In this paper, we propose to build multilayer coplanar waveguides (MCPW) using the multiple metal layers available in order to overcome the limitations of standard digital silicon technologies. 


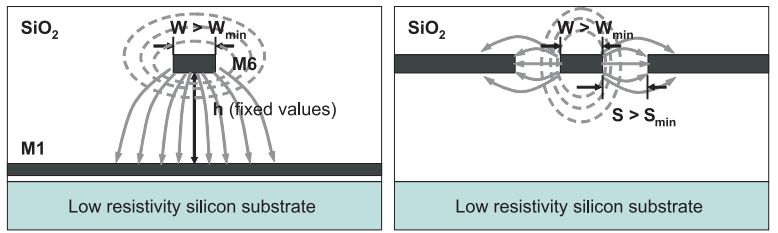

(a)

(b)

Fig. 2. (a) Microstrip and (b) CPW lines in silicon, showing field distribution and geometry limitations due to design rules (not to scale). The signal line is usually built on the top metal layer.

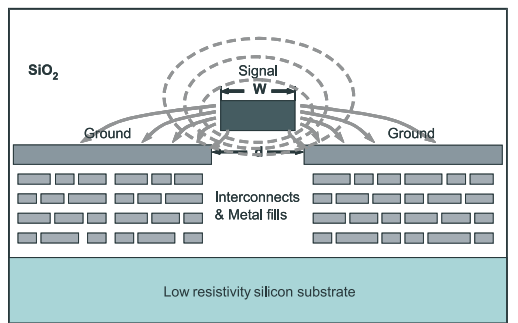

Fig. 3. Cross section of a MCPW, showing metal traces and fills underneath (not to scale). Note that the ground lines can be extended under the center strip.

\section{Silicon-Compatible Multilayer CPW}

Microstrips and coplanar waveguides (CPW) are commonly used planar transmission lines in silicon (Fig. 2). Both have relatively low loss, either because the metal ground plane shields the substrate in the case of microstrips, or because the electromagnetic field is largely moved away from the silicon substrate in CPWs. The characteristics impedance of a CPW can be designed by adjusting the spacing between signal and ground line, while that of a microstrip can be coarsely changed by chosen the metal layer for the ground plane. CPWs make it easier to connect transistors in layout, and tend to be more compact than microstrips. Hence, they are sometimes preferred to microstrips at microwave frequencies.

As discussed in Section I, layout design rules in silicon technologies make it difficult to achieve a wide range of characteristic impedance while maintaining low loss. In the microstrip case (Fig. 2-a), the distance between signal line and ground plane is limited, and so is the signal line width. Therefore, on-chip microstrips usually can only achieve relatively low impedance. For CPWs, the minimum impedance is limited by the slot width (metal spacing) allowed by design rules. The maximum impedance is limited partly by the center strip width (metal width) allowed by design rules, and partly because reducing the center strip width causes (a) large loss, and (b) characteristic impedance to become very sensitive to the stripwidth to slot-width ratio - any small process variations can cause significant change for impedance. It is also evident that any metal traces or fills on the metal layers below the signal lines will result in parasitic capacitance and change/degrade the characteristics of both transmission lines.
In order to overcome these limitations, we need to change the transmission line configuration. Starting from a microstrip, we can split the ground plane into two, and then by adjusting the gap between the two new "half ground planes" to increase the characteristic impedance. Starting from a CPW, we can move the ground lines to a lower metal layer, and thus remove the design rule restrictions on the slot width. Both approaches lead to the same multilayer $C P W$ (MCPW) configuration, as shown in Fig. 3. MCPWs can achieve a larger range of characteristic impedance than both microstrips and conventional CPWs by selecting the ground metal layer and changing the slot width. We can consider a microstrip and a CPW are just two special cases of a MCPW. Compared to conventional CPWs, MCPWs also relieve the current crowding at the edges of center conductor and ground lines, which helps to reduce conductor loss. MCPWs use different metal layers within the critical area, and other interconnects or metal fills can be placed underneath the ground lines. Therefore, it is much easier to meet the metal density requirements.

MCPWs have been previously proposed to reduce loss and achieve a large range of impedance in MMICs, and are also referred to as (center-)elevated CPW [4]-[7]. Most of these prior work focused on traditional MMIC technologies such as GaAs, and hence usually require additional process steps. In CMOS technologies, the readily-available multiple metal layers enable easier implementation and more configuration options.

\section{ANALYSis AND Simulation}

MCPWs in this work is designed based on process parameters from a commercial $0.18 \mu \mathrm{m}$ standard digital CMOS technology, which has $0.01 \Omega$-cm $\mathrm{P}+$ substrate and six metal layers. Top two metal layers are used to build the MCPWs. The signal line is on $2 \mu \mathrm{m}$ M6, and two ground lines are on $0.8 \mu \mathrm{m}$ M5. The width of the center strip is chosen to be $10 \mu \mathrm{m}$, the minimum metal width allowed by the design rule, in order to achieve as large characteristic impedance as possible. The length of the transmission lines is $512 \mu \mathrm{m}$. The design variable here is the ground spacing $d$. In practical design, other parameters can also be optimized. For example, multiple metal layers can be used for either the signal or ground lines.

Electromagnetic simulation based on Method of Moment [8] is carried out to analyze the characteristics of MCPWs. First, we studied their characteristic impedance $Z_{0}$. As shown in Fig. 4, when varying $d$ from $0 \mu m$ to $90 \mu m, Z_{0}$ changes from $14 \Omega$ to $95 \Omega$. Note that when $d=0 \mu \mathrm{m}$, the MCPW degenerates into a microstrip. When $d$ increases to over $60 \mu \mathrm{m}$, the increase of $Z_{0}$ slows down, since substrate coupling becomes dominant.

MCPW's propagation characteristics is then investigated. As shown in Fig. 5 (a), the effective dielectric constant decreases rapidly with frequency below $10 \mathrm{GHz}$, which is due to the slow-wave effect [9], [10], and then saturates at high frequencies when transmission lines enter the skin-effect mode. This mode change has strong dependency on the ground spacing $d$. When $d$ decreases from $100 \mu m$ to $20 \mu m$, because 


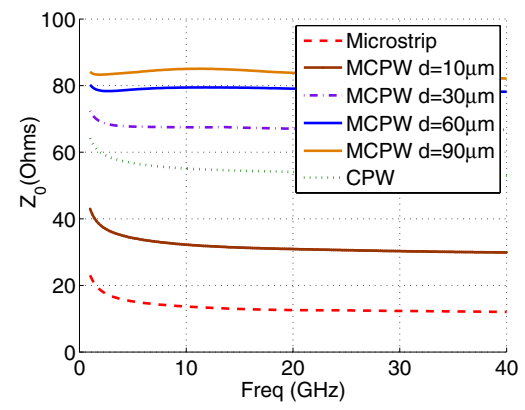

(a)

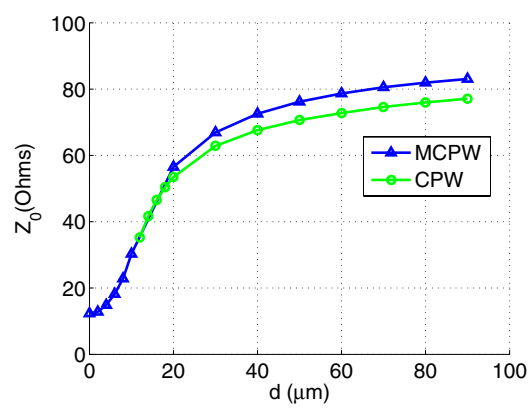

(b)

Fig. 4. Simulated characteristic impedance of MCPWs compared to microstrip and regular CPW. (a) frequency sweep; (b) with different ground spacing $d(30 \mathrm{GHz})$.

less electromagnetic field penetrates into the silicon substrate $\left(\epsilon_{S i}=11.9\right)$, the effective dielectric constant $\epsilon_{r, \text { eff }}$ decreases, as shown in Fig. 5 (b). When $d$ further decreases below $20 \mu \mathrm{m}, \epsilon_{r, e f f}$ increases again because the effect of finite metal thickness and conductivity becomes evident [11].

In order to keep the chip area small, the spacing between adjacent transmission lines should be as small as possible, which may cause parasitic coupling between lines. The coupling effect between two adjacent lines (see Fig. 6 (a)) on the same metal layers is shown in Fig. 6 (c) as a function of the separation between two lines. Even the separation $S$ is reduced to $10 \mu \mathrm{m}$, the coupling is still less than $-30 \mathrm{~dB}$ within $50 \mathrm{GHz}$ frequency range. Therefore, multiple MCPWs can run side-by-side with little coupling.

\section{Test Chip and Measurement Results}

A test chip with three MCPW lines (ground spacing $\mathrm{d}=0,30,60 \mu \mathrm{m}$ ) was fabricated using the aforementioned $0.18 \mu \mathrm{m}$ CMOS technology. The MCPW with $\mathrm{d}=0$ is in fact a microstrip line. A regular CPW line is also built on the same die for comparison. The chip photo is shown in Fig. 7. The MCPW and microstrip lines are built with M6 for signal line and M5 for ground. The regular CPW is built with M6 only. The ground spacing of the regular CPW is $20 \mu \mathrm{m}$. The

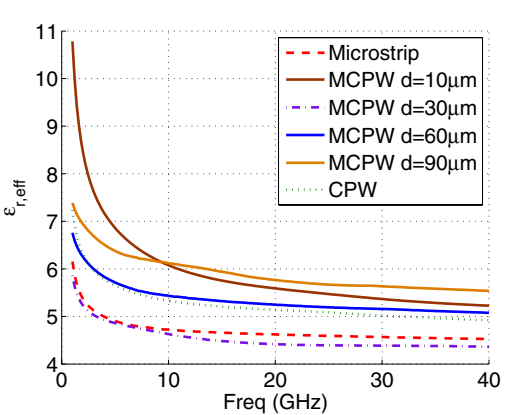

(a)

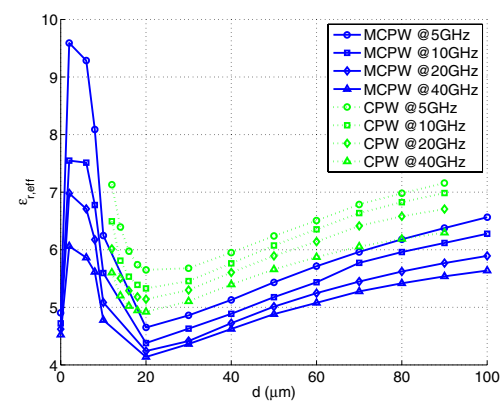

(b)

Fig. 5. Simulated effective dielectric constant of MCPWs compared to microstrip and regular CPW. (a) frequency sweep; (b) with different ground spacing $d$.

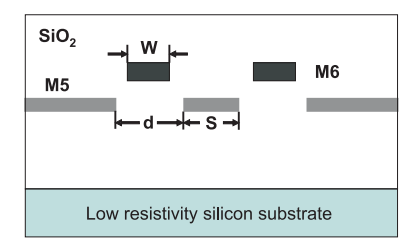

(a)

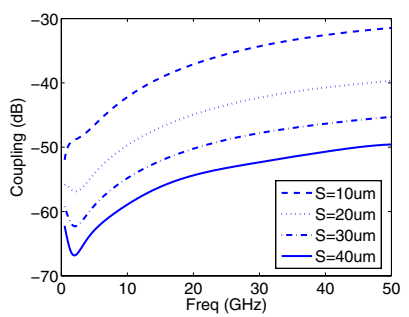

(b)
Fig. 6. Simulated MCPW coupling: (a) two adjacent MCPW lines built on M6 and M5 with $W=10 \mu m, d=18 \mu m$; (b) simulation results.

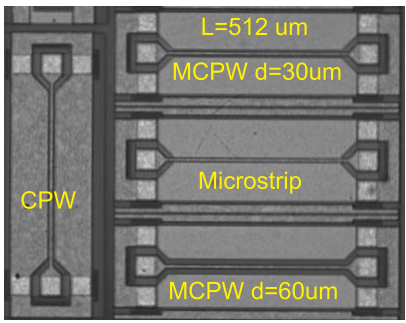

Fig. 7. Chip micrographs of the test chip. 


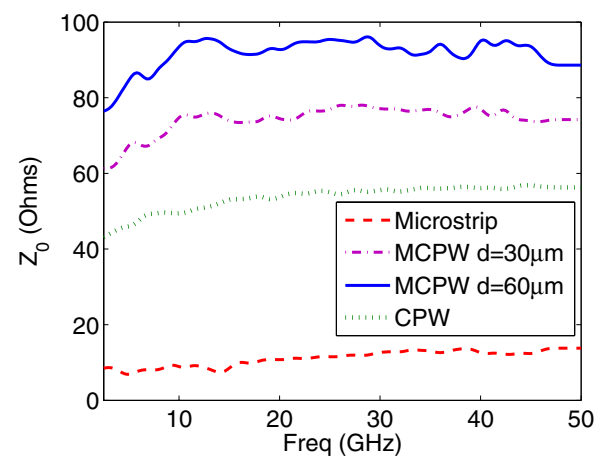

Fig. 8. Measured impedance.

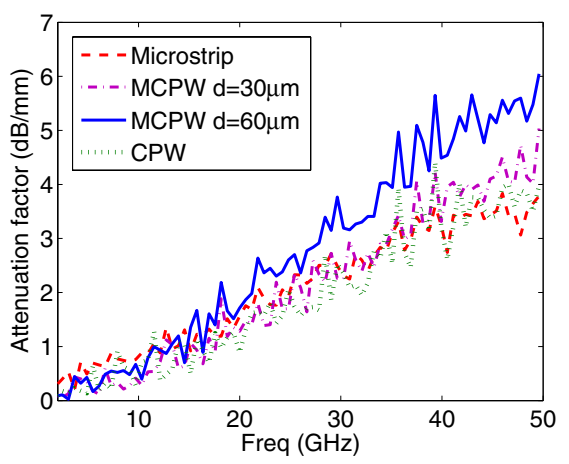

Fig. 9. Measured attenuation factor.

s-parameter measurement of MCPWs was performed using a vector network analyzer up to $50 \mathrm{GHz}$ via on-wafer probing. From measured s-parameters, characteristic impedance and propagation constant are extracted, and other transmission line parameters are calculated thereafter [12].

The measured characteristic impedance of the transmission lines are shown in Fig. 8, which spans from $10 \Omega$ for the microstrip to $90 \Omega$ for the MCPW with larger $d$. This verifies that a wide range of characteristic impedance can be achieved using the MCPW configuration. The results match well with simulation. The measured attenuation factor of the CPWs is shown in Fig. 9, which is about $1.8 \mathrm{~dB} / \mathrm{mm}$ at $20 \mathrm{GHz}$, and

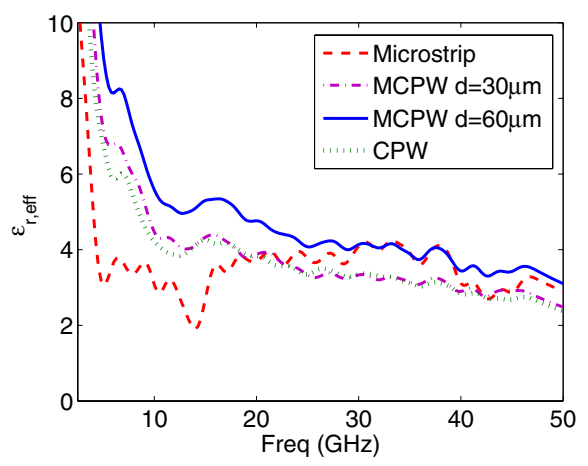

Fig. 10. Measured effective dielectric constant. increases with the frequency. Within $20 \mathrm{GHz}$, the MCPWs and microstrip have similar attenuation factor as normal CPW since the dominant loss mechanism is conductor loss. At higher frequencies, the MCPW with larger spacing shows larger attenuation because of the dielectric loss from silicon substrate. The measured effective dielectric constant is shown in Fig. 10, which has similar trend as simulation.

\section{CONCLUSION}

Multilayer CPW (MCPW) transmission lines can help to overcome the limitations of silicon technologies, including low substrate resistivity, minimum layout feature size, and minimum metal density requirements. In this work, characteristic impedance, dispersion and coupling of MCPWs were studied using full-wave electromagnetic simulation. A test chip was fabricated in a standard digital CMOS technology with low resistivity substrate. Measurement results show that MCPWs can achieve a wide range of characteristic impedance with loss comparable to normal CPWs.

\section{ACKNOWLEDGMENT}

The authors would like to thank P. Holloway, B. Chatterjee, J. Wan, B. Akinpelu, A. Shah and J. Yu of National Semiconductor for their help and support in chip fabrication. We also appreciate help from J. Zuegel and J. Marciante of Laboratory for Laser Energetics at University of Rochester.

\section{REFERENCES}

[1] International technology roadmap of semiconductor. www.itrs.org, 2005.

[2] B. Kleveland, T.H. Lee, and S.S. Wong. $50 \mathrm{GHz}$ Interconnect Design in Standard Silicon Technology. In IEEE Microwave Symposium, pages 1913-1916, 1998.

[3] Yunliang Zhu and Hui Wu. A $10-40 \mathrm{GHz} 7 \mathrm{~dB}$ Directional Coupler in Digital CMOS Technology. In IEEE Microwave Symposium, pages 1551-1554, 2006.

[4] M. Gillick and I.D. Robertson. Ultra Low Impedance CPW Transmission Lines of Multilayer MMICs. In IEEE Microwave Millimeter-Wave Monolithic Circuits Symp. Dig., pages 127-130, 1993.

[5] D. Budimir, Q.H. Wang, A.A. Rezazadeh, and I.D. Robertson. Vshape CPW transmission lines for multilayer MMICs. Electron. Lett., 31(5):1928-1929, Oct. 1995.

[6] F. Schnieder, R. Doerner, and W. Heinrich. High-impedance coplanar waveguides with low attenuation. IEEE Microw. Guided Wave Lett., 6(3):117-119, Mar. 1996

[7] Van Tuyen Vo, Lokesh Krishnamurthy, Qing Sun, and Ali A. Rezazadeh. 3-D Low-Loss Coplanar Waveguide Transmission Lines in Multilayer MMICs. IEEE Trans. Microwave Theory Tech., 54(6):2864-2871, Jun. 2006.

[8] In Sonnet User's Guide, 2006.

[9] Hideki Hasegawa, Mieko Furukawa, and Hisayoshi Yanai. Properties of Microstrip Line on Si-SiO2 System. IEEE Trans. Microwave Theory Tech., 19(11):869-881, Nov. 1971.

[10] Y. R. Kwon, V. M. Hietala, and K.S. Champlin. Quasi-TEM Analysis of "Slow-Wave" Mode Propagation on Coplanar Microstructure MIS Transmission Lines. IEEE Trans. Microwave Theory Tech., 35(6):545551, Jun. 1987.

[11] Jeng-Yi Ke and Chun Hsiung Chen. Dispersion and Attenuation Characteristics of Coplanar Waveguide with Finite Metallization Thickness and Conductivity. IEEE Trans. Microwave Theory Tech., 43(5):1128-1135, May 1995.

[12] W.R. Eisenstadt and Y. Eo. S-parameter based IC interconnect transmission line characterization. IEEE Trans. Compon., Hybrid, Manuf. Technol., 15(4):483-490, Aug. 1992. 